L'activité aux prises avec des systèmes ou dispositifs d'information

\title{
Innovation informationnelle et changements organisationnels : l'exemple de la traçabilité agroalimentaire informatisée.
}

Information Innovation and Organizational Change: The Case of Computerized Agri-food Traceability

André Salançon

\section{CpenEdition}

Journals

Édition électronique

URL : http://journals.openedition.org/edc/1118

DOI : 10.4000/edc. 1118

ISSN : 2101-0366

Éditeur

Université Lille-3

Édition imprimée

Date de publication : 1 décembre 2009

Pagination : 153-169

ISSN : 1270-6841

Référence électronique

André Salançon, «Innovation informationnelle et changements organisationnels : l'exemple de la traçabilité agroalimentaire informatisée. », Études de communication [En ligne], 33 | 2009, mis en ligne le 01 décembre 2011, consulté le 19 avril 2019. URL : http://journals.openedition.org/edc/1118 ; DOI : 10.4000/edc. 1118

Ce document a été généré automatiquement le 19 avril 2019

(C) Tous droits réservés 


\title{
Innovation informationnelle et changements organisationnels : l'exemple de la traçabilité agroalimentaire informatisée.
}

\author{
Information Innovation and Organizational Change: The Case of Computerized \\ Agri-food Traceability
}

André Salançon

Les préoccupations de sécurité des aliments ont conduit les pouvoirs publics à instituer ces dernières années une réglementation sur la traçabilité comme moyen de garantir la santé publique à travers des obligations nouvelles imposées aux acteurs des filières de production agroalimentaire ${ }^{1}$. Face à ces obligations, nombre d'entre eux ont fait appel aux TIC pour saisir, gérer et traiter efficacement la masse d'informations induite par la mise en œuvre de ces règlements. Ce faisant, l'utilisation des TIC dans ce contexte pose un certain nombre d'interrogations soulevées autant par la traçabilité que par les technologies elles-mêmes :

- 1 / Ces mesures conduisent en effet à redéfinir la fonction de logistique dans la chaîne de production - distribution - consommation : la disjonction des flux physiques et des flux d'informations place désormais la « ressource-information » au centre des jeux de relations entre les acteurs d'une filière.

- 2/ Le partage de l'information dans cette chaîne fait apparaître ensuite des débats sousjacents sur la question (au départ technique) de la normalisation de l'information et de l'interopérabilité nécessaire entre l'amont et l'aval qui s'invitent dans les rapports entre les acteurs.

- 3/ Enfin, la sécurité/traçabilité est porteuse de nombreux changements internes dans le fonctionnement des organisations. Elle fait émerger des processus d'adoption, d'apprentissage d'innovations et de changements avec lesquels les acteurs des filières tentent de composer. 
2 Le présent article rend compte de manière synthétique d'une étude de cas extraite d'une recherche menée dans la filière viticole, en l'occurrence six caves coopératives de la région Languedoc Roussillon ${ }^{2}$, qui a porté sur les corrélations existant entre l'adoption de Tic dans la mise en œuvre le la traçabilité et les changements dans les organisations. Elle a cherché, en particulier, à valider l'hypothèse selon laquelle celui qui détient les moyens de saisie, de gestion et de traitement des informations utilisées dans la réglementation traçabilité/sécurité des aliments, détient une partie du pouvoir susceptible de peser dans les relations internes à l'organisation ou externes avec ses fournisseurs ou ses clients et de favoriser ainsi les changements dans les relations entre acteurs.

\section{Une cave coopérative en prise avec la traçabilité informatisée}

Située dans la partie la plus au sud de la région, la cave coopérative «les vignerons du chardonnay ", créée en 1923, regroupe 270 coopérateurs dont le vignoble recouvre près de 1900 ha sur 12 communes. La production annuelle s'élève à 80000 hectolitres (50\% en vin d'Appellation d'Origine Contrôlée et $50 \%$ en vin de pays). Elle produit 2500000 bouteilles et 100000 bag in box par an pour le marché français qui représente (70\% du chiffre d'affaires) et l'exportation (30\% soit 1,5 million de bouteilles sur 3 continents (Asie, Amérique, Europe) et 25 pays) ${ }^{3}$.

4 Dans cette cave coopérative, le point de départ de la traçabilité se situe vers la fin des années 90 où qualité et sécurité alimentaires étaient devenues un sujet de préoccupation prioritaire pour les pouvoirs publics avec la promulgation des premiers règlements que les entreprises étaient invitées à appliquer ${ }^{4}$.

5 Plus récemment, avec l'obligation de traçabilité alimentaire, il s'est agi en fait de créer une pratique de traçabilité nouvelle; des formes de traçabilité papier avait toujours existé dans la cave mais sous des formes différentes qui atteignaient leur limite et peinaient à être efficaces face au développement quantitatif des informations à gérer avec la nouvelle règlementation.

6 Dès lors, la recherche d'efficacité entre autres conduisait les responsables de la cave coopérative à s'équiper, en 2003, d'outils informatiques pour répondre aux exigences réglementaires de la traçabilité ${ }^{5}$, accélérant ainsi l'informatisation de la cave déjà engagée de longue date autour d'un système centralisé AS 400.

7 Ce nouveau dispositif informatique engendrait la construction d'une base de données procurant une connaissance fine de chaque parcelle du vignoble permettant en interne une politique de plantation pour maitriser l'encépagement des vignobles des adhérents, lui-même déterminé par des objectifs commerciaux. Dans le même temps, cette connaissance fine des caractéristiques des parcelles par les viticulteurs, validées par le technicien de la cave, permettait de définir le classement de la parcelle qui joue par la suite sur la rémunération du viticulteur

8 Ainsi, cette base de données contient toutes les informations concernant chaque parcelle de vigne : modes de conduite, comptes-rendus de visites techniques, apports et analyses de l'état sanitaire des vendanges, taux de sucre, d'azote, de potassium etc qui donnent lieu à des analyses après la campagne pour améliorer la conduite de la vigne et la qualité des résultats au cours d'entretiens entre viticulteurs et techniciens de la cave. 
9 Si la traçabilité assistée par les Tic est aujourd'hui installée tant au sein de l'organisation de la coopérative que parmi les viticulteurs au moins en termes d'équipement, l'histoire de leur usage révèle une appropriation différenciée et loin d'être homogène et stabilisée. Tout au plus, elle montre le poids que la modernité fait peser sur les pratiques des utilisateurs et laisse entrevoir les enjeux sous jacents.

10 A bien des égards en effet, la traçabilité assistée par les Tic dans cette cave coopérative constitue un changement dans les pratiques et les représentations qui peuvent s'y rattacher. Au demeurant, l'efficacité de l'outil informatique pour la gestion des cuves, pour le pilotage des travaux de la cave et son contrôle a progressivement contribué à faire admettre son utilité à l'intérieur de la cave en tant qu'unité de production du vin.

11 Au-delà du gain en termes d'efficacité fonctionnelle et individuelle, les avis positifs sur les bénéfices du dispositif (base de données et logiciels partagés en réseau) se concentrent sur deux points principaux :

- 1/ la gestion de la cave : « Aujourd'hui, tout est centralisé. En cinq minutes, je sors l'état de la régie, j'ai mes bilans par qualité, par client, par appellation, par millésime. » AS, directeur de production

- 2/ la capacité de l'organisation à répondre tout autant à des exigences réglementaires qu'à des demandes de la part de la clientèle, en particulier celles des centrales d'achat qui par « effet domino » ont conduit les responsables à engager des changements à l'intérieur de l'organisation.

Face à une nouvelle crise économique du secteur viti-viticole, une réaction vigoureuse s'imposait de la part des producteurs et de leurs organisations. Elle passait par une remise en question des conceptions et des méthodes résumée par le président de la cave coopérative : "On avait des invendus, des produits qui ne correspondaient pas aux attentes ou des concurrences qui étaient trop fortes et qui faisaient que ces produits là n'avaient plus de place sur le marché. Donc il fallait changer les choses et adapter la production aux ventes...il fallait partir de la bouteille et remonter à la parcelle. Voilà, ça c'était la grande idée ».R.T, président de la cave.

\section{Des innovations qui suscitent des réactions mitigées et changent les positions des acteurs.}

Cependant, l'une des difficultés principales du changement induit dans la mise en oeuvre de la traçabilité assistée par les Tic a été la perception différenciée de son intérêt pour les principales catégories d'acteurs concernés.

\section{Les opérateurs et techniciens de la cave}

Même si la saisie des données ou l'enregistrement des informations est une opération partagée « décentralisée » entre plusieurs personnes (3 à 4 pour la traçabilité amont, ${ }^{6}$ ou 7 pour la partie cave et conditionnement-expédition), même si la mise en réseau est une fonctionnalité importante dans la configuration de cette cave coopérative, ces facteurs ne semblent pas modifier fondamentalement le mode d'organisation du travail des techniciens de la cave.

Certes, ce nouveau dispositif constitue une facilité et un confort dans le travail : " pour moi c'est une facilité. D'être... De faire un peu moins marcher ma mémoire. J'ai toutes les informations sur le logiciel. Pour travailler les mouvements, je m'en sers beaucoup ». XC, 
caviste vin blanc. En revanche, sa pratique apparaît davantage profiter au responsable de la production qui supervise les données saisies par les personnels de cave et qui suit par la même occasion le travail de chaque opérateur.

Cette analyse en fait va de pair avec une peur de remise en question des habitudes de travail qui étaient rôdées parfois depuis plus de vingt ans d'expérience. A cette crainte, se sont ajoutés dès le départ des erreurs et des dysfonctionnements qui auraient pu conduire les salariés à renoncer.

L'arrivée de cette technologie a révélé des dysfonctionnements techniques en particulier dans une première période où des logiciels de traçabilité et autres matériels (Pockets PC) étaient en phase de tests et d'essais qui se soldaient par des avis réservés sur les outils à cause des dysfonctionnements, d'une grande exigence de temps pour s'approprier l'outil, voire d'une inadaptation de l'outil aux pratiques.

Pour les opérateurs de la cave, un point paraît cependant central dans cette phase d'appropriation technologique : le sentiment de perte d'autonomie par rapport à un système de traçabilité avec des fiches- papier sur les cuves dans la mesure où :

"Ces fiches, c'est nous qui les gérions... chacun marque ce qu'il fait, comment il le fait, donc. On peut, s'il y a une erreur, on peut mettre le doigt sur l'erreur et sur la personne qui l'a faite., avec les fiches peut-être aussi, mais c'est pas pareil. C'est... Ça fait moins peur, c'est moins inquisiteur, c'est . Là, ça fait un peu Big Brother... Tandis que là, l'outil informatique il est suivi par toute la cave C'est-à-dire, par exemple, mon chef, il peut regarder ce qu'on a fait de chez lui...Traçabilité c'est ça, en fait. C'est pour surveiller. Même si c'est la traçabilité, on surveille en même temps, on surveille un peu les autres. Je pense que c'est un facteur qui fait peur aussi...Je pense que sur la question ici, ça ne joue pas trop. Je pense que sur certaines structures, ça peut, ça dépend du caractère de ceux qui dirigent. Ça peut vraiment être un outil de pression ». XC, caviste vin blanc

Ces différentes résistances faisaient que les cavistes ont maintenu pendant deux ans une double traçabilité papier et logicielle aujourd'hui abandonnée, le temps que l'un des cavistes fasse aux autres la preuve de son intérêt. Ainsi, la traçabilité assistée par les Tic serait entrée dans les pratiques ordinaires et serait même devenue désormais une « habitude».

\section{Les viticulteurs-coopérateurs}

Dans la partie amont de la chaîne c'est à dire chez les viticulteurs, la mise en place autrefois des politiques de qualité, de sécurité et plus récemment de traçabilité a modifié assez sensiblement les pratiques.

21 La traçabilité à la parcelle est essentiellement établie par contrat entre chaque viticulteur et la cave. Ce contrat oblige les viticulteurs à fournir à la cave les traitements effectués dans les parcelles (fin juin et mi-août) de manière à vérifier le respect des délais de traitements phytosanitaires avant récolte qui conditionnent les dates des vendanges.

Dans ce cadre très strict, la coopérative a essayé de mettre en place des logiciels de saisie chez les viticulteurs, sans succès : «On a relevé cinq personnes quoi. Fiasco. On a essayé, l'année dernière, un poste de saisie ici. Où ils saisissaient directement chez nous. Ça n'a pas marché non plus. Bon, là, on va essayer autre chose. Avec Géodasia[société éditrice de logiciels de traçabilité], on va voir si on peut trouver, si on peut trouver d'autres solutions ». RM, technicien viticole. 

acteur redouté par les professionnels de ce milieu en raison de l'incidence fiscale que comporte cette activité. Comme pour ce responsable qualité, d'aucuns s'accordent sur l'opinion selon laquelle «Le premier élément de traçabilité au départ, c'est la répression des fraudes. C'était pas la sécurité alimentaire ». P.M., responsable qualité et informatique.

Depuis peu cependant avec la mise en place de la traçabilité s'est opéré un changement important dans les responsabilités imparties à ce service: nul ne doute que la concentration de l'information sur un support informatique, traitant les données de 
façon rationnelle, facilite leur accès aux services administratifs chargés de contrôler l'application de la sécurité et de la traçabilité alimentaire.

\section{Les éditeurs de logiciels et autres prestataires informatiques}

31 Depuis l'ancienne informatique centralisée sur des machines relativement importantes avec des applications dédiées à la gestion comptable, la cave a connu le progrès technologique apporté par la micro-informatique. Cette évolution a entraîné l'apparition d'une nouvelle catégorie d'acteurs, en l'occurrence de jeunes sociétés informatiques qui se sont lancées dans des développements logiciels adaptés à la nouvelle configuration technologique. Cette action collective a privilégié une relation avec une jeune société régionale avec laquelle la cave a cherché à innover avec les risques que cela comportait.

Elle poursuit aujourd'hui cette coopération avec un projet qui vise à accroître l'efficacité du dispositif tout en maîtrisant les coûts de fonctionnement. Des marges de progrès sont également reconnues nécessaires pour optimiser et affiner les saisies d'informations mais surtout mieux exploiter le volume d'informations déjà collectées.

\section{Quand les TIC favorisent la construction d'un nouvel espace de médiations.}

En conclusion de cette brève présentation d'une étude de cas, et en élargissant l'analyse aux autres cas étudiés, on peut affirmer que, de manière générale, les applications de traçabilité assistée par les TIC ont eu pour effet principal de faire intervenir l'information dans les activités des entreprises de manière plus importante. Elles génèrent divers changements dans les relations entre les acteurs au sein d'une même organisation ainsi qu'au sein d'une filière. Plus particulièrement, l'utilisation des TIC dans la mise en œuvre de la réglementation sur la sécurité/traçabilité des aliments valide l'hypothèse sur deux points :

\section{Les TIC augmentent le pouvoir des caves sur les coopérateurs}

L'utilisation des TIC augmente le pouvoir de «l'unité de production - commercialisation du vin » sur les « viticulteurs - adhérents » qui produisent les raisins et qui tendraient à devenir les sous-traitants de l'unité de production - commercialisation du vin.

Dans ce processus de mise en oeuvre de la réglementation relative à la sécurité/ traçabilité des aliments, les caves apparaissent de prime abord comme les acteurs principaux au centre de ce processus. En tant qu'unité de production et de transformation du raisin, soucieuse de garantir la qualité des raisins et la traçabilité des matières premières transformées, elles ont développé une fonction majeure de créateur de base de données (saisie et gestion) qui les ont conduites à étendre leur pouvoir sur les viticulteurs-coopérateurs : elles orientent l'encépagement des parcelles, propriétés des viticulteurs-coopérateurs, elles dictent les procédures à respecter pour améliorer la qualité de la production, elles organisent les apports...

Dans ces différentes actions, la production d'information s'accompagne de la mise en place d'une normalisation des informations exigée à la fois par le support technologique (TIC) mais aussi par les différents cahiers des charges entrés en vigueur dans la même 
période. Les enregistrements selon des grilles prédéfinies et surtout contrôlées par différentes instances créent nécessairement une pratique nouvelle dans les relations aussi bien individuelles que collectives que les caves entretiennent avec les coopérateurs qui sont en l'occurrence leurs fournisseurs de matières premières. L'activité informationnelle devient ainsi un enjeu important dans la mesure où elle est devenue ou devient une activité autour de laquelle se structurent les relations fournisseurstransformateurs qui sont à la base de l'activité de production vinicole. Les difficultés rencontrées dans la mise en place de ces règlements qui induisent des changements dans les pratiques et gestes professionnels ne diminuent en rien la logique de rationnalisation des modes opératoires sous jacente qui visent à accroître les performances des dispositifs techniques et organisationnels.

Elles apportent la preuve à contrario que l'outillage informationnel qui se met en place provoque des modifications à divers échelons inter dépendants depuis la pratique individuelle du métier jusqu'à l'organisation collective de la transformation des matières premières, ce que désigne le terme de changement organisationnel.

\section{Le pouvoir des caves coopératives est subordonné à celui des négociants et distributeurs} réglementation sur la sécurité/traçabilité des aliments par les caves ne donne pas à ces dernières un ascendant suffisant sur cette clientèle. Le pouvoir des caves est en effet subordonné aux exigences de la clientèle qui joue, par ailleurs, de manière variable et opportuniste avec les règlementations sanitaires dans les négociations commerciales.

Cette fonction structurante de l'information induisant un pouvoir des caves sur leurs adhérents qui sont leurs principaux fournisseurs, se trouve dans le même temps subordonnée à l'influence et au pouvoir direct exercé par les clients qui font intervenir la traçabilité en sus des règles de négociation commerciale tout comme, dans un autre registre, les instances administratives du contrôle de leur activité. L'information concernant la traçabilité saisie, traitée et gérée par les caves avec l'assistance des TIC, rentre ainsi dans le jeu des relations entre les acteurs principaux de la filière au point qu'on peut considérer qu'elle structure leurs positions et qu'elle agit sur la dynamique des interactions qui se produisent entre eux selon des stratégies spécifiques et parfois contradictoires. Les producteurs affichent une stratégie qui consiste à orienter leurs actions vers la satisfaction des clients, contrairement à une culture vigneronne inverse longtemps dominante. Cette nouvelle pratique induit des modifications dans la manière de conduire le vignoble, d'exploiter le résultat des vendanges, d'élaborer un vin et de valoriser la production.

Sur fond de crise de la surproduction, les clients tendent à occuper de plus en plus le champ de la production en intégrant la traçabilité comme arme commerciale bien que celle-ci ne soit pas d'un usage systématique et rigide. En effet pour les négociants et la distribution, le prix demeure la variable qui souvent l'emporte dans leur décision d'achat en deçà des exigences réglementaires de traçabilité, à l'exception des achats pour l'étranger pour lesquels le respect des normes de traçabilité est strict. Dans cette nouvelle orientation d'action caractérisée par un pilotage de la production par l'aval, la traçabilité assistée par les TIC devient un élément de réassurance du distributeur qui trouve dans la mise en œuvre de ce dispositif en quelque sorte des raisons de faire commerce en 
reportant la responsabilité du risque sanitaire sur le producteur ou l'importateur sur la base des informations saisies, gérées et traitées par ces derniers. A contrario, le fait d'avoir pour le producteur une banque de données parfaitement maîtrisée aussi bien du côté matériel que celui des données représente un gage de crédibilité et de force que les caves peuvent exploiter, sachant que la distribution demeure cependant en grande partie maitre du jeu puisque ce sont les achats qu'elle fait qui donnent tout leur sens aux actions que les organisations mettent en place.

\section{Les TIC révélatrices des relations complexes entre acteurs d'une filière}

Limitée aux seules entreprises coopératives viticoles de la région Languedoc-Roussillon et aux usages des TIC dédiés à la seule application de traçabilité, cette recherche ne prend tout son sens que si l'on rattache l'apparition des TIC à des pratiques d'information soustendues par des logiques de normalisation, de rationnalisation enchevêtrées dans le jeu des relations entre acteurs au sein d'une organisation ainsi qu'entre les organisations d'une filière. Ces dernières ne sont pas étrangères à des mutations en cours dans la filière qui consistent à inverser le schéma de production établi en adoptant le concept « De la bouteille à la grappe de raisin et non l'inverse ". L'analyse des pratiques décrites par les acteurs interrogés conduit, en effet, à dégager une conception nouvelle de la production à l'opposé de celle de la culture vigneronne dans laquelle le client-consommateur avait à s'adapter au vin produit par les viticulteurs. Depuis quelques décennies et notamment du fait de la crise de la sur-production de vin notamment en Europe, le modèle s'est inversé : il est aujourd'hui assez largement convenu que la demande de la consommation pilote la production ou en tout cas il est proclamé que le sort des producteurs est lié à un changement de conception de l'activité vini-viticole.

Ce pilotage par l'aval dans lequel les dispositifs informationnels interviennent s'appuie en effet sur deux sous-ensembles d'acteurs, négociants et distributeurs d'un côté, organismes certificateurs et de contrôle de l'autre dont la présence et l'action viennent renforcer la pression sur les caves, agissent sur les changements organisationnels du fait notamment de l'utilisation croissante des dispositifs informationnels.

En premier lieu, les négociants et distributeurs - désignés par l'appellation distribution sont des acteurs intégrés depuis longtemps dans la filière viti-vinicole. Les négociants pour lesquels le prix constituait le critère principal d'achat ont été pendant longtemps les clients principaux des caves qui leur vendaient principalement leur vin en vrac qu'ils se chargeaient de conditionner. Le développement de la grande distribution a permis à cette dernière de se constituer en client principal des caves notamment de celles qui conditionnaient elles-mêmes leur vin. A partir de la période où la qualité s'est imposée aux producteurs, la distribution a profité de cette pratique pour formuler des exigences qui, par ailleurs, ont tendu les relations mais lui a permis de formuler de nouvelles exigences au moment de l'obligation de traçabilité.

Ainsi ces deux vagues de réglementations (d'abord qualité puis sécurité/traçabilité alimentaire) et les batteries de moyens de communication utilisés, tous supports confondus, constituent le socle en quelque sorte sur lequel l'organisation de la production et donc celle des producteurs vont se restructurer : désormais l'objectif des producteurs consiste à satisfaire les désirs des acheteurs principaux (négociants / centrales d'achat) ; toutes sortes de stratégies et de moyens sont orientés dans ce sens parmi lesquels la 
rationalisation des méthodes de production et de gestion assistée par les Tic. Dans cette perspective, les Tic parallèlement au flux de matières canalisent les flux d'informations engendrés par les mesures de traçabilité, permettent de les gérer et de les traiter pour répondre aux exigences de la demande.

En second lieu, les organismes certificateurs et cabinets d'audit d'une part et les services de contrôle administratif (DGCCRF : direction générale de la concurrence, de la consommation et de la répression des fraudes) d'autre part dont les interventions sont spécifiques, forcent au respect des lois et règlements et s'appliquent à exercer un contrôle strict.

Dans un contexte de crise, les relations commerciales avec la distribution et à fortiori avec les acheteurs étrangers sont en effet régies en grande partie par des normes de qualité et de traçabilité spécifiques qui vont parfois au-delà des réglementations en vigueur et qui engendrent contrôles et vérifications particuliers par des organismes certificateurs et par des audits faisant peser sur les producteurs une menace commerciale permanente. Dans ces actions de contrôle, l'usage des tic - essentiellement base de données - permet de satisfaire aux demandes immédiates et construit à plus long terme une image positive de la coopérative si elle se montre capable de résoudre un problème d'identification de l'origine d'un produit qui serait défaillant.

Cependant, la vérification de l'application des réglementations par le service des fraudes rentre dans une longue histoire de relations tendues et conflictuelles avec les milieux professionnels viticoles. Généralement redoutés en raison des incidences fiscales, les contrôles des agents de l'administration portant sur la sécurité sanitaire et sur la traçabilité des produits se trouvent en quelque sorte facilités par le traitement informatique des informations jusque là dispersées sur plusieurs supports papier. Cette situation nouvelle incitant les caves à maintenir un double système de saisie et de stockage de l'information sur support papier et informatique. Au-delà des raisons avancées de sécurité des informations, la maîtrise du système d'information demeure un enjeu de contrôle qui ne peut se concevoir indépendamment de la contrainte du contrôle obligatoire et potentiel, étant entendu qu'en outre, il revient à l'organisation contrôlée de faire la preuve qu'elle respecte les lois et règlements ce qui n'est pas sans effet sur les conduites et les pratiques relationnelles.

En tout état de cause, la traçabilité des objets ne peut être dissociée d'une réelle inter action entre ce dispositif règlementaire et les relations entre les acteurs qui demeurent dynamisées par les stratégies permanentes de pouvoirs. Si l'information peut être considérée selon un certain point de vue comme un acteur à part entière, l'adoption et la maîtrise des technologies d'information créent un espace de médiations des pouvoirs que se disputent les acteurs déployant par ailleurs leur propre stratégie.

Au-delà de la filière viti-vinicole, on peut élargir les résultats de cette analyse à d'autres filières de production dans la mesure où des logiques quasi similaires sont à l'œuvre :

- une logique économique marquée par une concentration croissante des acteurs et canaux de distribution entraînant une adaptation obligée des producteurs même si des alternatives de commercialisation émergent,

- une logique de rationnalisation des process de production engageant une recherche d'efficacité et de gain de productivité,

- une logique de contrôle social allant au-delà du domaine de santé publique justifiant la réglementation sur la sécurité / traçabilité. 
Ainsi, il est démontré (une nouvelle fois) que le recours aux TIC s'inscrit dans des rapports de forces entre acteurs d'une filière de production, que leur insertion dans les entreprises répond à des intérêts stratégiques, que l'information dans les milieux professionnels prend une place déterminante dans les changements organisationnels. Cette étude de cas révèle également l'intérêt de cet angle d'approche pour apprécier la question des effets des TIC sur le développement économique des entreprises, question qui demeure un objet de recherche à approfondir compte tenu des mutations en cours.

\section{BIBLIOGRAPHIE}

Agreste, (2002), Agreste Languedoc Roussillon. Recensement 2001 des caves coopératives : diversité des stratégies et des résultats économiques. Octobre 2002 Draf L-R 12 p.

Besnard, Sonia, Biré, Chantal et Victor, Patrice, (2008), L'intégration des Tic est encore incomplète dans les entreprises Insee. Première $\mathrm{N}^{\circ} 1184$ - avril 2008

Bernoux, Philippe, (2004), La sociologie du changement dans les entreprises et les organisations. Le Seuil. 2004. 307 p.

Boutary, Martine, (2003), TIC et PME : des usages aux stratégies L'Harmattan 2003, 203 p.

Brousseau, Eric, Rallet, Alain, (1999), Technologies de l'information, organisation et performances économiques $350 \mathrm{p}$.

Callon, Michel, (1986), Eléments pour une sociologie de la traduction : la domestication des coquilles Saint Jacques et des marins-pêcheurs dans la baie de Saint Brieuc. L'année Sociologique. Numéro Spécial 1986 pp169-208.

Crozier, Michel, Friedberg, Erhard, (1977), L'acteur et le système. Les contraintes de l'action collective. Paris. Seuil. 500 p.

Fabbe-Costes, Nathalie, Lemaire, Christelle, (2001), « La traçabilité totale d'une supply chain : principes, obstacles et perspectives de mise en œuvre ", Revue française de Gestion Industrielle, Vol 20, $\mathrm{n}^{\circ} 3$, pp 23-52

Friedberg, Erhard, (1993), Le pouvoir et la règle. Dynamique de l'action organisée. Paris. Seuil. 404 p. Guyot, Brigitte, (2004), Eléments pour une approche informationnelle dans les organisations. Sciences de la société $n^{\circ} 63$ octobre $2004 \mathrm{p}$ 11-25.

Hermitte, Marie Angèle, (2003), La traçabilité des personnes et des choses. Précautions, pouvoirs et maîtrise In Traçabilité et responsabilité sous la dir. Philippe Pedrot Economica 2003 pp 1-34.

Laporte, Jean- Pierre, Touzard, Jean-Marie, (2004), La grande transformation d'un vignoble de masse. AgroLine mars $2004 \mathrm{n}^{\circ} 32$.

Miège, Bernard, (1995), La pensée communicationnelle. Grenoble. PUG. 118 p.

Miège, Bernard, (1996), La société conquise par la communication : tome 1 Logiques sociales. PUG. 226 p. 
Miège Bernard, (2007), La société conquise par la communication : tome 3, Les Tic entre innovation technique et ancrage social. PUG. 235 p.

Nanni, Elisabetta, Salançon, André, (2005), La traçabilité : définition, réglementation, objectifs, technologies, Série Etudes n 08-2005. UMR Moisa 50 p.

Renard, Aurélie, (2005), Diagnostic des systèmes et outils traçabilité » dans les filières céréales/ oléoprotéagineux et plantes aromatiques. Esitpa, Acta, Actia. 48 p +annexes.

Salançon, André, (2003), Nouvelles technologies de l'information et de la communication et développement des entreprises agroalimentaires : le cas du Languedoc-Roussillon Montpellier UMR MOISA, 2005 Série Etudes ; nº 07.

Violette, Justine, (2005), Etude de la traçabilité dans la filière vin Acta, ITV, Actia 19 p + annexes.

\section{NOTES}

1. La plus significative de cette mouvance règlementaire est le règlement (CE) N.178/2002 qui établit les principes généraux et les prescriptions générales de la législation alimentaire incluant des précisions relatives aux domaines d'applications et aux responsabilités qui incombent aux exploitants du secteur alimentaire.

2. Changements organisationnels, traçabilité et Tic. A.Salançon, B.Fallery, P.Baillette, N.Rahali. UMR Moïsa /Cregor. Série Etudes mars 200965 p.

3. Les six caves coopératives enquêtées participent des caractéristiques des 360 coopératives dénombrées en 2001 en région Languedoc Roussillon, rassemblant 50000 adhérents, cultivant $71 \% \mathrm{du}$ vignoble régional ( $214000 \mathrm{ha}$ ), produisant presque les trois quarts de la récolte régionale (près de 15 millions d'hl) avec un chiffre d'affaires autour de 1000 millions d'euros, employant une quantité de travail évaluée à 3000 équivalents temps plein (coopératives, unions et filiales) (Agreste 2002).

4. En l'occurrence, un décret français daté du 28 mai 1997, consécutif à une directive européenne (93 43 de 1993) est ici la référence mise en avant pour situer le véritable début de l'histoire de la traçabilité dans cette structure.

5. Il s'agit de deux logiciels :GeoVINI, développé au départ par la société Géodasea qui en 2007 a fusionné avec une autre filiale d'Isagri Vitarea, commercialisant désormais ce produit. Ce logiciel permet d'enregistrer les mouvements de vins dans la cave à partir de l'apport des raisins et avant la mise en bouteille. C'est en résumé le logiciel traçabilité-cave qui inclut tous les intrants introduits dans l'élaboration des vins. Il est l'outil principal des techniciens et responsables des caves rouge et blanc.WinCoop est un autre logiciel développé par une structure locale, émanation de la coopération viticole régionale Informatique Conseil Service, spécialisée dans la filière vin.

6. GeoTRACE fait partie d'un pack Vignoble GeoCOOP-GeoTRACE véritable système d'information géographique dédié à la filière viticole et développé par la société Vitaréa qui «garantit la traçabilité amont avec des solutions de collecte d'information adaptée ».

7. IFS : référentiel privé appartenant à la Fédération du Commerce et de la distribution en France et à HDE en Allemagne (P.M., responsable qualité et informatique).

8. DGCCRF : Direction Générale de la concurrence, de la concurrence et de la répression des fraudes. 


\section{RÉSUMÉS}

L'utilisation de Tic dans la mise en œuvre de la réglementation sur la traçabilité en agroalimentaire met en exergue la place et le rôle de l'information dans le fonctionnement des entreprises. Extraite d'une série d'investigations dans la filière vitivinicole, une étude de cas montre les effets de cette double insertion - réglementaire et technologique - dans les organisations économiques du fait de la place grandissante de l'information dans les nouvelles normes de production.

The use of information technology (IT) in the implementation of food industry traceability regulations highlights the impact of information on business functions. Drawn from research conducted in the wine industry, this case study shows the effects of this two-fold development, at once regulatory and technological, on the commercial entities involved. It concludes that IT is playing an ever-increasing role in new production norms within business organizations.

\section{INDEX}

Mots-clés : TIC, changement organisationnel, traçabilité, normalisation, informatique, innovation, commerce

Keywords : ICT, organizational change, traceability, normalization, computer science, innovation, trade

\section{AUTEUR}

\section{ANDRÉ SALANÇON}

André Salançon est ingénieur de recherche du ministère de l'agriculture. Au sein de Montpellier Sup Agro et plus particulièrement au sein du département des sciences économiques sociales et de gestion, il initie les élèves-ingénieurs agronomes à la sociologie des organisations, des controverses socio-techniques et à celle des médias.

Intégré dans l'Unité Mixte de Recherche Moïsa (marché, organisations, institutions et stratégies d'acteurs) depuis une dizaine d'années, il centre ses travaux de recherche sur l'insertion des TIC dans les organisations professionnelles agricoles et les industries agroalimentaires.

salancon@supagro.inra.fr 